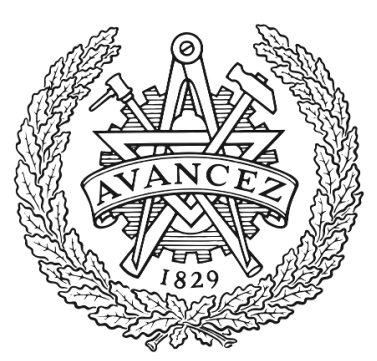

CHALMERS

UNIVERSITY OF TECHNOLOGY

\title{
Asymmetric Complex-Vector Models with Application to VSC-Grid Interaction
}

Downloaded from: https://research.chalmers.se, 2023-04-26 13:18 UTC

Citation for the original published paper (version of record):

Harnefors, L., Wang, X., Chou, S. et al (2020). Asymmetric Complex-Vector Models with Application to VSC-Grid Interaction. IEEE Journal of Emerging and Selected Topics in Power Electronics, 8(2): 1911-1921. http://dx.doi.org/10.1109/JESTPE.2020.2972070

N.B. When citing this work, cite the original published paper.

C2020 IEEE. Personal use of this material is permitted.

However, permission to reprint/republish this material for advertising or promotional purposes 


\title{
Asymmetric Complex-Vector Models With Application to VSC-Grid Interaction
}

\author{
Lennart Harnefors $^{\circledR}$, Fellow, IEEE, Xiongfei Wang ${ }^{\circledR}$, Senior Member, IEEE, Shih-Feng Chou ${ }^{\circledR}$, Member, IEEE, \\ Massimo Bongiorno ${ }^{\circledR}$, Senior Member, IEEE, Marko Hinkkanen ${ }^{\circledR}$, Senior Member, IEEE, \\ and Mikko Routimo ${ }^{(}$, Member, IEEE
}

\begin{abstract}
The properties of complex space-vector models for asymmetric three-phase systems are investigated in this article. Most importantly, three alternative methods for the stability analysis of the asymmetric closed-loop systems are presented. The end results avoid the usage of matrix manipulations. It is shown how the theory can be applied to modeling and stability analysis of a grid-connected voltage-source converter (VSC). The methods are compared using numerical examples.
\end{abstract}

Index Terms-Complex space vectors, complex transfer functions, converter control, passivity, stability.

\section{INTRODUCTION}

$\mathbf{S}$ YMMETRIC (balanced) three-phase dynamic systems can be modeled using complex space vectors together with complex transfer functions [1]. This allows a single-input single-output (SISO) notation, even though the system in reality is multi-input-multi-output (MIMO), since each space vector has two components.

Conversely, the input admittance of a grid-connected voltage-source converter (VSC), as seen from the point of common coupling (PCC), has, in general, asymmetric (also called unsymmetric or imbalanced) properties [2]. This traditionally necessitates MIMO models using real space vectors and $2 \times 2$ transfer function matrices [3], [5]. Such modeling methods are established well [6], [9], yet, they add complexity relative to SISO models. For stability analysis, the conventional Nyquist criterion (NC) is replaced by the generalized

Manuscript received October 21, 2019; revised January 14, 2020; accepted February 2, 2020. Date of publication February 6, 2020; date of current version May 6, 2020. This work was supported in part by ABB. Recommended for publication by Associate Editor Jon Are Suul. (Corresponding author: Lennart Harnefors.)

Lennart Harnefors is with $\mathrm{ABB} \mathrm{AB}$, Corporate Research, 72178 Västerås, Sweden (e-mail: lennart.harnefors@se.abb.com).

Xiongfei Wang and Shih-Feng Chou are with the Department of Energy Technology, Aalborg University, 9100 Aalborg, Denmark (e-mail: xwa@et.aau.dk; shc@et.aau.dk).

Massimo Bongiorno is with the Department of Electrical Engineering, Chalmers University of Technology, 41296 Göteborg, Sweden (e-mail: massimo.bongiorno@chalmers.se).

Marko Hinkkanen is with the Department of Electrical Engineering and Automation, Aalto University, 02150 Espoo, Finland (e-mail: marko.hinkkanen@aalto.fi).

Mikko Routimo is with ABB Oy Drives, 00380 Helsinki, Finland (e-mail: mikko.routimo@fi.abb.com).

Color versions of one or more of the figures in this article are available online at http://ieeexplore.iee.org.

Digital Object Identifier 10.1109/JESTPE.2020.2972070
NC (GNC) [10], [11]. Matrix modeling of the grid impedance is needed, which is inconvenient particularly for a symmetric grid, where an SISO model suffices.

Steps toward generalizing the complex-vector theory to asymmetric models, including VSC modeling and analysis, have been taken. In [1], it is shown how a $2 \times 2$ transfer function matrix can be decomposed into two complex transfer functions, respectively, for the (here called) symmetric and antisymmetric parts. An asymmetric complex-vector SISO model is obtained. In [12], it is suggested to neglect the antisymmetric part. Avoiding this simplification, an MIMO scheme is considered in a series of articles by Rygg et al. [13], [14] and Zhang et al. [15], [16]. The scheme in [13] is called modified sequence domain, but is here, for consistency with the aforementioned SISO model, called an asymmetric complex-vector MIMO model. Compared with the real-vector MIMO model, certain benefits are gained, including the usage of SISO techniques for stability analysis and grid-impedance modeling. A similar model, but in the stationary $\alpha \beta$ frame rather than in the synchronous $d q$ frame, is proposed in [17].

In this article, further results for the asymmetric complexvector SISO and MIMO models are presented in Section II. They include expressions for cascading two SISO models and certain properties of the generic transfer function matrix of the MIMO model. The method for modeling of the grid-connected VSCs in [12] is revisited and clarified concerning the antisymmetric part. In addition, the principles in [2] for the dissipation and passivity of an MIMO model are briefly reprised. It is shown that the measure of passivity, here called the passivity index, can be conveniently expressed in the transfer functions of the asymmetric complex-vector SISO model.

The most important contribution is the consideration in Section III of three alternative methods for closed-loop stability analysis using the asymmetric complex-vector models. Method 1 extends the approach in [12] by considering also the antisymmetric part. The closed-loop system is separated into a symmetric inner loop and an antisymmetric outer loop. Stability is analyzed by applying the NC to both loops. (Another method using cascaded loops for stability analysis of grid-connected VSCs - but not relying on complex vectors-is proposed in [18] and [19].) Methods 2 and 3, respectively, use the eigenvalue and determinant GNC variants applied to the 
asymmetric complex-vector MIMO model. Expressions that solely use the transfer functions of the corresponding SISO model are derived, thereby obviating matrix manipulations. Methods 2 and 3 are close in spirit to the SISO approach for stability analysis in [15], but they are generic for any asymmetric system, whereas in [15], the interconnection of two impedances is considered. Although grid-connected VSCs are the foremost application, there are potentially many other, such as subsynchronous-oscillation studies [20].

In Section IV, finally, the three methods for stability analysis of Section III are compared using illustrative numerical examples (one of them verified experimentally), as applied to the VSC model of Section II. It is verified that all methods give identical results concerning stability versus instability, while they have different properties regarding how the information is presented. Whereas Method 2 may be the most straightforward to use, Method 1 provides valuable insight into the mechanisms causing instability and the risk thereof. Method 3 is found to be less desirable, since the resulting Nyquist curve often is difficult to interpret, and can tentatively be dismissed from further studies.

\section{Complex-Vector Modeling OF ASYMMETRIC SYSTEMS}

Complex space vectors and their associated complex transfer functions (denoted by bold letters) facilitate the modeling of the symmetric systems. Assuming modeling in the $d q$ frame, with the generic input and output vectors $\mathbf{u}=u_{d}+j u_{q}$ and $\mathbf{y}=y_{d}+j y_{q}$ and the generic transfer function $\mathbf{G}(s)=G_{d}(s)+j G_{q}(s)$, we have ${ }^{1}$

$$
\mathbf{y}=\mathbf{G}(s) \mathbf{u} \text {. }
$$

Despite that each vector accounts for two signals, the $d$ and $q$ components, an SISO notation is facilitated. By resolving the $d$ and $q$ components, the MIMO correspondence to (1) is obtained, using two-row real space vectors and a $2 \times 2$ transfer function matrix (denoted by italic letters) as

$$
\underbrace{\left[\begin{array}{c}
y_{d} \\
y_{q}
\end{array}\right]}_{y}=\underbrace{\left[\begin{array}{ll}
G_{d d}(s) & G_{d q}(s) \\
G_{q d}(s) & G_{q q}(s)
\end{array}\right]}_{G(s)} \underbrace{\left[\begin{array}{c}
u_{d} \\
u_{q}
\end{array}\right]}_{u}
$$

with

$$
G_{d d}(s)=G_{q q}(s)=G_{d}(s) \quad G_{q d}(s)=-G_{d q}(s)=G_{q}(s) .
$$

By lifting the restriction (3), allowing four unique matrix elements, asymmetric systems - such as VSCs and salient-pole synchronous machines - can be modeled as well. However, then, (1) no longer suffices, which precludes modeling using complex vectors and an SISO notation.

\section{A. Asymmetric Complex-Vector SISO Model}

In [1], it is proposed to extend the SISO model (1) to asymmetric systems by using two parts, here called symmetric and antisymmetric, each having its own transfer function. The antisymmetric part takes the conjugated input signal, denoted

\footnotetext{
${ }^{1}$ The Laplace variable $s$ shall be considered as the operator $s=d / d t$, where appropriate.
}

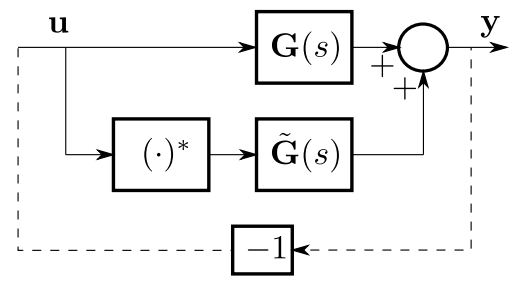

(a)

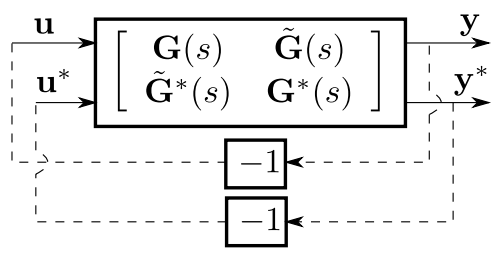

(b)

Fig. 1. (a) Asymmetric complex space-vector SISO model. (b) Corresponding MIMO model. The dashed lines show optional feedback loops that are considered in Section III.

by $(\cdot)^{*}$. With a cleaner notation than in [1], in the sense that subscripts are avoided, the model is expressed as

$$
\mathbf{y}=\mathbf{G}(s) \mathbf{u}+\tilde{\mathbf{G}}(s) \mathbf{u}^{*} .
$$

The block diagram in Fig. 1(a) illustrates (4) graphically (the dashed feedback loop is, at this stage, disregarded). With $\tilde{\mathbf{G}}(s)=\tilde{G}_{d}(s)+j \tilde{G}_{q}(s)$ in addition to $\mathbf{G}(s),(4)$ has four degrees of freedom. In [21], it is demonstrated how this model can be applied to various asymmetric systems.

The effect of the conjugation made in the antisymmetric part is illustrated particularly well for a steady-state frequency component in $\mathbf{u}$, which rotates counterclockwise, say $\mathbf{U} e^{j \omega t}$. The conjugation gives rise to a mirror component $\mathbf{U}^{*} e^{-j \omega t}$, rotating clockwise. Filtered through $\tilde{\mathbf{G}}(s)$, the mirror component adds to the output signal.

The model (4) can be extended in various ways. Of particular importance is the cascade connection of two asymmetric systems, e.g., asymmetric admittance and impedance

$$
\begin{aligned}
\mathbf{i} & =\mathbf{Y}(s) \mathbf{u}+\tilde{\mathbf{Y}}(s) \mathbf{u}^{*} \\
\mathbf{y} & =\mathbf{Z}(s) \mathbf{i}+\tilde{\mathbf{Z}}(s) \mathbf{i}^{*} .
\end{aligned}
$$

Substituting (5) in (6) yields

$$
\mathbf{y}=\mathbf{Z}(s)\left[\mathbf{Y}(s) \mathbf{u}+\tilde{\mathbf{Y}}(s) \mathbf{u}^{*}\right]+\tilde{\mathbf{Z}}(s)\left[\mathbf{Y}(s) \mathbf{u}+\tilde{\mathbf{Y}}(s) \mathbf{u}^{*}\right]^{*}
$$

which simplifies to (4) with

$$
\begin{aligned}
\mathbf{G}(s) & =\mathbf{Z}(s) \mathbf{Y}(s)+\tilde{\mathbf{Z}}(s) \tilde{\mathbf{Y}}^{*}(s) \\
\tilde{\mathbf{G}}(s) & =\mathbf{Z}(s) \tilde{\mathbf{Y}}(s)+\tilde{\mathbf{Z}}(s) \mathbf{Y}^{*}(s) .
\end{aligned}
$$

In a similar fashion, the input-output description of any asymmetric system or circuit, no matter how complex, can be reduced to the SISO model (4).

Remark 1: In the sequel, some equations explicitly involve the $d$ and $q$ components of a complex transfer function. They can be extracted according to [1]

$$
G_{d}(s)=\frac{\mathbf{G}(s)+\mathbf{G}^{*}(s)}{2} \quad G_{q}(s)=\frac{\mathbf{G}(s)-\mathbf{G}^{*}(s)}{2 j} .
$$


Remark 2: Observe that, when conjugating a complex transfer function as in (10), $s$ is to be considered real. This means that care must be exercised when computing the frequency response of a conjugated transfer function, e.g., $\mathbf{G}^{*}(j \omega)$. Numerically, conjugation is more conveniently applied to the full frequency response, denoted as $[\mathbf{G}(j \omega)]^{*}$. From the rules of conjugation, we have that $\mathbf{G}^{*}(s)=\left[\mathbf{G}\left(s^{*}\right)\right]^{*}$, and specifically, for $s=j \omega$

$$
\mathbf{G}^{*}(j \omega)=[\mathbf{G}(-j \omega)]^{*} .
$$

This is straightforward to implement, e.g., in MATLAB. A vector representing $\mathbf{G}(j \omega)$ is first computed, with points symmetrically distributed for positive and negative $\omega$. The vector representing $[\mathbf{G}(-j \omega)]^{*}$ is then obtained by applying the commands fliplr and conj.

\section{B. Asymmetric Complex-Vector MIMO Model}

Suppose that there is a mirror component already in the input signal. This is the case particularly when closing the feedback loop in Fig. 1(a) (see Section III). The SISO model (4) then does not immediately reveal the couplings between the two components. To facilitate this, an extension to a corresponding MIMO model can be made by first conjugating (4) as $\mathbf{y}^{*}=\mathbf{G}^{*}(s) \mathbf{u}^{*}+\tilde{\mathbf{G}}^{*}(s) \mathbf{u}$. The asymmetric complex-vector MIMO model (denoted by bold-italic letters) is then formed by combining (4) and its conjugate, collecting the original and conjugated (mirror) components in two-row vectors as [see Fig. 1(b)]

$$
\underbrace{\left[\begin{array}{l}
\mathbf{y} \\
\mathbf{y}^{*}
\end{array}\right]}_{\boldsymbol{y}}=\underbrace{\left[\begin{array}{cc}
\mathbf{G}(s) & \tilde{\mathbf{G}}(s) \\
\tilde{\mathbf{G}}^{*}(s) & \mathbf{G}^{*}(s)
\end{array}\right]}_{\boldsymbol{G}(s)} \underbrace{\left[\begin{array}{c}
\mathbf{u} \\
\mathbf{u}^{*}
\end{array}\right]}_{\boldsymbol{u}} .
$$

Models in the form (12) are extensively studied in [13]-[16]. Among other things, it is shown in [13] that $\boldsymbol{G}(s)$ can be obtained from $G(s)$ of the original real-vector MIMO model (2) by the linear transformation

$$
\boldsymbol{G}(s)=\boldsymbol{T} G(s) \boldsymbol{T}^{-1}, \quad \boldsymbol{T}=\frac{1}{\sqrt{2}}\left[\begin{array}{rr}
1 & j \\
1 & -j
\end{array}\right] .
$$

$\boldsymbol{T}$ is unitary, i.e., $\boldsymbol{T}^{-1}=\boldsymbol{T}^{H}$, where the superscript $H$ indicates the transpose-conjugate (Hermitian conjugate). Transformation (13), although with the matrix $\boldsymbol{T} \rightarrow \boldsymbol{T} / \sqrt{2}$, was introduced already in the mid-1970s [20].

Some benefits of (12) relative to (2) are stated in [13] and [16], among them that the off-diagonal elements of $\boldsymbol{G}(s)$ quantify the level of asymmetry. For the special case of a symmetric system, they vanish, reducing (12) to (1) and its conjugate.

Another benefit of (12) is that $\boldsymbol{G}(s)$ has a restricted structure. Whereas the elements of $G(s)$ in (2) can be arbitrary, and the diagonal and off-diagonal elements of $\boldsymbol{G}(s)$ are identical, but conjugated. This fact is pointed out in [22], but it does not seem to be fully embraced in the literature. The reason for the restriction is that the information of the SISO model is duplicated in the MIMO model; the second row of (12) is the conjugate of the first row. The asymmetric complexvector SISO and MIMO models are, therefore, complementary

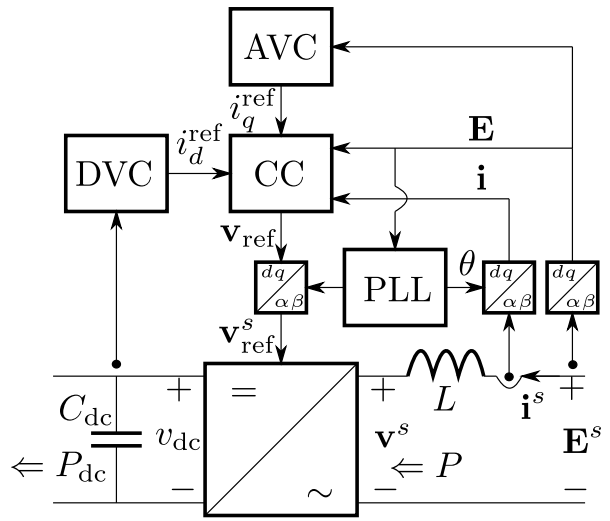

Fig. 2. VSC circuit diagram and control system.

and carry exactly the same information. The main purpose of the MIMO model is as a tool for calculating the results that cannot be obtained using the SISO model, as exemplified in the sequel.

As (12) is generic for any asymmetric system, it is not surprising that the restricted structure of $\boldsymbol{G}(s)$ is invariant under the basic matrix manipulations. For adding or subtracting two matrices, this is obvious. For $\boldsymbol{G}(s)=\boldsymbol{Z}(s) \boldsymbol{Y}(s)$, it follows from (5) and (6) that (8) and (9) are the resulting elements of $\boldsymbol{G}(s)$. Inverting a matrix in the form (12), e.g., to obtain the admittance corresponding to an impedance, yields

$$
\boldsymbol{G}^{-1}(s)=\left[\begin{array}{cc}
\mathbf{G}_{-1}(s) & \tilde{\mathbf{G}}_{-1}(s) \\
\tilde{\mathbf{G}}_{-1}^{*}(s) & \mathbf{G}_{-1}^{*}(s)
\end{array}\right]
$$

where $\mathbf{G}_{-1}(s)=\mathbf{G}^{*}(s) /\left[\mathbf{G}(s) \mathbf{G}^{*}(s)-\tilde{\mathbf{G}}(s) \tilde{\mathbf{G}}^{*}(s)\right]$ and $\tilde{\mathbf{G}}_{-1}(s)=-\tilde{\mathbf{G}}(s) /\left[\mathbf{G}(s) \mathbf{G}^{*}(s)-\tilde{\mathbf{G}}(s) \tilde{\mathbf{G}}^{*}(s)\right]$. Finally, transposing a matrix in the form (12) gives $\tilde{\mathbf{G}}(s) \rightarrow \tilde{\mathbf{G}}^{*}(s)$ and corresponds to trading places between the blocks $(\cdot)^{*}$ and $\tilde{\mathbf{G}}(s)$ in Fig. 1(a).

\section{Application to Modeling of Grid-Connected VSCs}

A grid-connected VSC can be modeled as an asymmetric input admittance, as observed from the PCC [2], [12]. From the control laws that normally are used for the grid-connected VSCs, the admittance parts $\mathbf{Y}(s)$ and $\tilde{\mathbf{Y}}(s)$ can be derived without intermediately using a real-vector MIMO model (as done in [2]). This modeling closely follows [12]. Yet, there is the need for clarification, since in [12], attention is paid only to the derivation of $\mathbf{Y}(s)$, not $\tilde{\mathbf{Y}}(s)$ (see the Appendix for more details).

1) Control-System Structure: The VSC control system under consideration is depicted in Fig. 2. The ac-side circuit is referred to the $\alpha \beta$ frame-denoted by the superscript $s-$ whereas the control system is implemented in the $d q$ frame. The quantities correspond to $\mathbf{i}^{s}=e^{j \theta} \mathbf{i}$, and so on, where $\theta$ is the $d q$-frame angle and $\mathbf{i}$ is the converter input current. The converter input filter is approximated as purely inductive, with inductance $L$. Four control loops are incorporated: the current controller (CC), the phase-locked loop (PLL), the directvoltage controller (DVC), and the alternating-voltage controller (AVC). 
The CC is given by the control law

$$
\mathbf{v}_{\text {ref }}=\mathbf{F}_{c}(s)\left(\mathbf{i}-\mathbf{i}_{\mathrm{ref}}\right)-j \omega_{1} L \mathbf{i}+\mathbf{H}(s) \mathbf{E}
$$

where $\mathbf{v}_{\text {ref }}$ is the reference for the converter voltage $\mathbf{v}$, the two related by the linear model $\mathbf{v}=\mathbf{G}_{l}(s) \mathbf{v}_{\text {ref }}$ for the converter latency [24], $\mathbf{i}_{\mathrm{ref}}=i_{d}^{\mathrm{ref}}+j i_{q}^{\mathrm{ref}}$ is the reference for $\mathbf{i}$, term $-j \omega_{1} L \mathbf{i}$ is the $d q$ decoupler, and $\omega_{1}$ is the fundamental angular frequency. Unlike [12], but similar to [2], feedforward through the low-pass filter $\mathbf{H}(s)$ [with $\mathbf{H}(0)=1$ ] of the PCC voltage $\mathbf{E}$ is included. A proportional $(\mathrm{P})$ controller $\mathbf{F}_{c}(s)$ is the core to which an integral (I) part and/or resonant parts can be added, as desired.

The PLL computes the $d q$-frame angle as

$$
\theta=\frac{1}{s}\left[F_{p}(s) \operatorname{Im}\{\mathbf{E}\}+\omega_{1}\right]
$$

where a PI controller $F_{p}(s)$, possibly cascaded with a low-pass filter to suppress disturbances such as harmonics, is generally employed.

The DVC controls the direct (dc-link) voltage $v_{\mathrm{dc}}$ by the dc-link energy $W_{\mathrm{dc}}=\left(C_{\mathrm{dc}} / 2\right) v_{\mathrm{dc}}^{2}$, where $C_{\mathrm{dc}}$ is the dc-link capacitance, using the control law

$$
i_{d}^{\mathrm{ref}}=F_{d}(s)\left(W_{\mathrm{dc}}^{\mathrm{ref}}-W_{\mathrm{dc}}\right) .
$$

Controller $F_{d}(s)$ is often structurally similar, perhaps even identical, to $F_{p}(s)$.

The AVC controls the PCC-voltage magnitude by the law

$$
i_{q}^{\mathrm{ref}}=F_{a}(s)\left(E_{0}-|\mathbf{E}|\right)
$$

where $E_{0}$ is the PCC-voltage magnitude reference. $F_{a}(s)$ may be a PI controller, possibly purely $\mathrm{P}$ or purely I, depending on the situation. A low-pass filter can-or, rather, should-be included in cascade, particularly if a $\mathrm{P}$ part is used.

2) Input Admittance: From the control laws (15)-(18), expressions for the input admittance parts $\mathbf{Y}(s)$ and $\tilde{\mathbf{Y}}(s)$ can be derived by adding up the individual contributions, as detailed in the Appendix. The result is

$$
\begin{aligned}
& \mathbf{Y}(s)=\mathbf{Y}_{c}(s)+\mathbf{Y}_{p}(s)+\mathbf{G}_{c}(s) \mathbf{Y}_{d}(s)+\mathbf{Y}_{a}(s) \\
& \tilde{\mathbf{Y}}(s)=-\mathbf{Y}_{p}(s)+\mathbf{G}_{c}(s) \mathbf{Y}_{d}^{*}(s)+\mathbf{Y}_{a}(s)
\end{aligned}
$$

where

$$
\begin{aligned}
\mathbf{G}_{c}(s) & =\frac{\mathbf{G}_{l}(s) \mathbf{F}_{c}(s)}{s L+j \omega_{1} L\left[1-\mathbf{G}_{l}(s)\right]+\mathbf{G}_{l}(s) \mathbf{F}_{c}(s)} \\
\mathbf{Y}_{c}(s) & =\frac{1-\mathbf{G}_{l}(s) \mathbf{H}(s)}{s L+j \omega_{1} L\left[1-\mathbf{G}_{l}(s)\right]+\mathbf{G}_{l}(s) \mathbf{F}_{c}(s)} \\
\mathbf{Y}_{p}(s) & =-\frac{1}{2}\left[\mathbf{Y}_{c}(s)-\mathbf{G}_{c}(s) \mathbf{i}_{0} / E_{0}\right] g_{p}(s) \\
\mathbf{Y}_{d}(s) & =-\frac{1}{2}\left[\mathbf{Y}_{c}(s)+\mathbf{i}_{0}^{*} / E_{0}\right] g_{d}(s) \\
\mathbf{Y}_{a}(s) & =-j \frac{1}{2} \mathbf{G}_{c}(s) F_{a}(s) .
\end{aligned}
$$

Here, $\mathbf{G}_{c}(s)$ and $\mathbf{Y}_{c}(s)$ are, respectively, the closed-loop system and the input-admittance contribution of the CC. Equations (23)-(25) quantify the input-admittance contributions of the PLL, DVC, and AVC, respectively. In (23) and (24), $g_{p}(s)$ and $g_{d}(s)$ are, respectively, the closed-loop systems of the PLL and the DVC, given as

$$
g_{p}(s)=\frac{E_{0} F_{p}(s)}{s+E_{0} F_{p}(s)} \quad g_{d}(s)=\frac{\kappa E_{0} F_{d}(s)}{s+\kappa E_{0} F_{d}(s)}
$$

where $\kappa=3 /\left(2 K^{2}\right)$ and $K$ is the space-vector scaling constant. ${ }^{2}$ Normally, the controllers are designed so that the bandwidths of $g_{p}(s)$ and $g_{d}(s)$ are substantially lower than that of $\mathbf{G}_{c}(s)$.

In (19) and (20), it can be noted that, being symmetric, the $\mathrm{CC}$ affects only $\mathbf{Y}(s)$. Neglecting the latency, i.e., $\mathbf{G}_{l}(s)=1, \mathbf{G}_{c}(s)$ and $\mathbf{Y}_{c}(s)$ both become real for a real $\mathbf{F}_{c}(s)$. This property is fundamental for reducing the complexity of the real MIMO models, since a diagonal admittance matrix with relatively simple expressions is obtained [2]. Complex models obviate this issue; a complex $\mathbf{F}_{c}(s)$-resulting, e.g., from the addition of the reduced-order generalized integrators [12] - poses no further difficulties.

Conversely, the PLL, DVC, and AVC, having an asymmetric impact, affect both $\mathbf{Y}(s)$ and $\tilde{\mathbf{Y}}(s)$.

Remark 3: Equations (19) and (20) are simplified under certain, but often realistic, assumptions. First, since $\mathbf{G}_{c}(s)$ has high bandwidth, $\mathbf{G}_{c}(s) \approx 1$ within the much lower bandwidths of $g_{p}(s)$ and $g_{d}(s)$. This allows putting $\mathbf{G}_{c}(s)=1$ in (19), (20), and (23). Second, converters are often operated at or near unity power factor, allowing $i_{q 0}=0$ to be assumed. Third, the PLL and DVC closed-loop bandwidths are often selected in the same range. Therefore, with the final assumption that $g_{d}(s)=g_{p}(s)$, we get $\mathbf{Y}_{p}(s)=-\left[\mathbf{Y}_{c}(s)-i_{d 0} / E_{0}\right] g_{p}(s) / 2$ and $\mathbf{Y}_{d}(s)=-\left[\mathbf{Y}_{c}(s)+i_{d 0} / E_{0}\right] g_{p}(s) / 2$, giving

$$
\begin{aligned}
& \mathbf{Y}(s)=\mathbf{Y}_{c}(s)\left[1-g_{p}(s)\right]+\mathbf{Y}_{a}(s) \\
& \tilde{\mathbf{Y}}(s)=-\left(i_{d 0} / E_{0}\right) g_{p}(s)+\mathbf{Y}_{a}(s) .
\end{aligned}
$$

A considerable simplification is obtained. It can be noted that $\mathbf{Y}(s)$ becomes operating-point-independent, because the PLL and DVC contributions that are proportional to $i_{d 0}$ cancel in (19), whereas in (20), they add constructively.

\section{Passivity Properties and Passivity Index}

Studying the passivity (dissipative) properties of an asymmetric system is particularly useful for the input admittance of a grid-connected VSC [2]. For $Y(s)$, in the real-vector MIMO model (2), they are quantified by the eigenvalues $p_{Y}(\omega)$ of $A(\omega)=(1 / 2)\left[Y(j \omega)+Y^{H}(j \omega)\right]$ [23]. If the minimum eigenvalue $p_{Y}^{\min }(\omega)$-here called the passivity index ${ }^{3}-$ is nonnegative, $Y(s)$ is dissipative for that $\omega$. From (13), it follows that $Y(j \omega)+Y^{H}(j \omega)=\boldsymbol{T}^{-1}\left[\boldsymbol{Y}(j \omega)+\boldsymbol{Y}^{H}(-j \omega)\right] \boldsymbol{T}$. Consequently, for the complex-vector MIMO model, ${ }^{4}$ the passivity index is found as the minimum eigenvalue of $\boldsymbol{A}(\omega)=$ $(1 / 2)\left[\boldsymbol{Y}(j \omega)+\boldsymbol{Y}^{H}(-j \omega)\right]$, whose elements are given as

$$
\boldsymbol{A}(\omega)=\frac{1}{2}\left[\begin{array}{c}
\mathbf{Y}(j \omega)+\mathbf{Y}^{*}(-j \omega) \tilde{\mathbf{Y}}(j \omega)+\tilde{\mathbf{Y}}(-j \omega) \\
\tilde{\mathbf{Y}}^{*}(j \omega)+\tilde{\mathbf{Y}}^{*}(-j \omega) \mathbf{Y}^{*}(j \omega)+\mathbf{Y}(-j \omega)
\end{array}\right] .
$$

${ }^{2} \kappa=1$ for power-invariant scaling $(K=\sqrt{3 / 2})$ or normalization to per-unit quantities.

${ }^{3}$ Note the difference relative to [23], where the passivity index is instead defined as the ultimate minimum $\min _{\omega} p_{Y}(\omega)$.

${ }^{4} \mathrm{~A}$ way of calculating the passivity index for the complex-vector SISO model is yet to be found. 


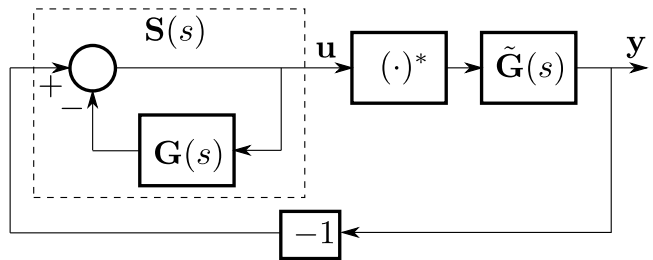

Fig. 3. Closed-loop system in Fig. 1(a) redrawn as a symmetric inner loop and an antisymmetric outer loop.

After simplification, the following expression in the components of $\mathbf{Y}(s)$ and $\tilde{\mathbf{Y}}(s)$ is obtained:

$$
\begin{aligned}
& p_{Y}^{\min }(\omega)=\operatorname{Re}\left\{Y_{d}(j \omega)\right\} \\
&-\sqrt{\left[\operatorname{Im}\left\{Y_{q}(j \omega)\right\}\right]^{2}+\left[\operatorname{Re}\left\{\tilde{Y}_{d}(j \omega)\right\}\right]^{2}+\left[\operatorname{Re}\left\{\tilde{Y}_{q}(j \omega)\right\}\right]^{2}} .
\end{aligned}
$$

From the standpoint of closed-loop stability (see Section III), it is generally beneficial if the regions of negative passivity index are as narrow as possible [2]. In this respect, (30) is instructive, as it shows explicitly that only the $d$ component of the symmetric part, more specifically the real part thereof, can contribute to a positive passivity index. All other parts can only contribute negatively.

Remark 4: Equations (27) and (28) are illustrative concerning the VSC input-admittance passivity properties under the assumptions stated in Remark 3. They show that only the $\mathrm{CC}$, by $\mathbf{Y}_{c}(s)$, can contribute to a positive passivity index. The minus sign of $g_{p}(s)$ in (27) indicates that the PLL and the DVC contribute negatively, increasingly so with a higher bandwidth [2]. As $\mathbf{G}_{c}(s)$ is predominantly real, $\mathbf{Y}_{a}(s)$ is predominantly imaginary and, thus, adds to $Y_{q}(s)$ and $\tilde{Y}_{q}(s)$, implying a negative contribution from the AVC. Somewhat different properties are obtained when $g_{d}(s) \neq g_{p}(s)$, on the other hand. For example, (23) and (24) indicate that, by making the bandwidth of $g_{p}(s)$ much larger than that of $g_{d}(s)$, a positive contribution in the rectifier mode $\left(i_{d 0}>0\right)$ can be obtained. Conversely, the opposite selection of the bandwidths gives a positive contribution in the inverter mode $\left(i_{d 0}<0\right)$.

\section{Closed-Loop Stability Analysis}

By including the dashed lines in Fig. 1, a closed-loop system is obtained. This is a generic model for any asymmetric system that uses negative feedback, but the grid-connected VSCs are the foremost application. Three alternative stability analysis methods are considered in this section.

\section{A. Method 1 (Two-Loop Representation of the SISO Model)}

Fig. 1(a) can be redrawn as two cascaded feedback loops, as shown in Fig. 3. These loops are mathematical abstractions rather than physical control loops, but they allow the stability impacts of $\mathbf{G}(s)$ and $\tilde{\mathbf{G}}(s)$ to be analyzed separately. Closing the symmetric inner loop forms the sensitivity function [11]

$$
\mathbf{S}(s)=\frac{1}{1+\mathbf{G}(s)} .
$$

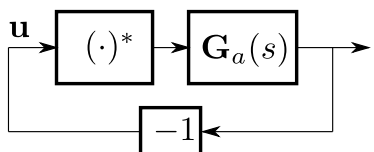

(a)

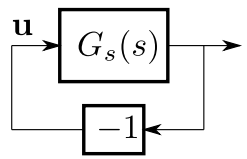

(b)
Fig. 4. (a) Antisymmetric outer loop with $\mathbf{G}_{a}(s)=\tilde{\mathbf{G}}(s) \mathbf{S}(s)$. (b) Equivalent symmetric outer loop with $G_{s}(s)=-\mathbf{G}_{a}(s) \mathbf{G}_{a}^{*}(s)$.

The stability of (31) can obviously be evaluated by applying the $\mathrm{NC}$ to the return ratio $\mathbf{G}(s)$. For a complex transfer function, the response for $\omega>0$ is not generally the conjugate of that for $\omega<0$. Consequently, evaluation for $s=j \omega$ over $-\infty<\omega<\infty$ is required, giving a Nyquist curve, which generally is not symmetric about the real axis.

The antisymmetric outer loop can be further simplified as shown in Fig. 4(a), giving $\mathbf{u}=-\mathbf{G}_{a}(s) \mathbf{u}^{*}$, where

$$
\mathbf{G}_{a}(s)=\tilde{\mathbf{G}}(s) \mathbf{S}(s)=\frac{\tilde{\mathbf{G}}(s)}{1+\mathbf{G}(s)} .
$$

Conjugating $\mathbf{u}=-\mathbf{G}_{a}(s) \mathbf{u}^{*}$ yields $\mathbf{u}^{*}=-\mathbf{G}_{a}^{*}(s) \mathbf{u}$, and, thus, by eliminating $\mathbf{u}^{*}$ among the two relations, $\mathbf{u}=\mathbf{G}_{a}(s) \mathbf{G}_{a}^{*}(s) \mathbf{u}$. That is, the antisymmetric outer loop can be resolved into a symmetric loop, as illustrated in Fig. 4(b). The stability of this loop can be assessed by applying the $\mathrm{NC}$ to the return ratio

$$
G_{s}(s)=-\mathbf{G}_{a}(s) \mathbf{G}_{a}^{*}(s) .
$$

Note that $\mathbf{G}_{a}(s) \mathbf{G}_{a}^{*}(s)$ has real coefficients, which is why $G_{s}(s)$ does not have bold-letter notation.

Method 1 offers some valuable insight. Poor stability margins of $\mathbf{G}(s)$ give a small minimum distance from the Nyquist curve to the critical point -1 , the inverse being the sensitivity peak $|\mathbf{S}(j \omega)|_{\max }$. Since $\mathbf{S}(s)$ appears as a factor in $G_{s}(s)$, the sensitivity peak expands the Nyquist curve of $G_{s}(s)$, likely reducing its stability margins. Thus, if $\mathbf{G}(s)$ and $\tilde{\mathbf{G}}(s)$ can be shaped, the primary focus should be the stability margins of $\mathbf{G}(s)$, since they affect both loops. Examples are given in Section IV.

Remark 5: If $\mathbf{G}(s)$ and $\tilde{\mathbf{G}}(s)$ both are stable, the total closedloop system is guaranteed to be asymptotically stable if the Nyquist curves for $\mathbf{G}(s)$ and $G_{s}(s)$ both avoid encirclement of -1 . This requirement is conservative, however, as there may be cases where the inner loop is unstable [i.e., the Nyquist curve for $\mathbf{G}(s)$ encircles -1 clockwise], but the total system is stabilized by the outer loop [i.e., the Nyquist curve for $G_{s}(s)$ encircles -1 counterclockwise]. An example thereof is given in Section IV.

\section{B. Method 2 (Eigenvalue GNC Variant Applied to the MIMO Model)}

By considering the MIMO model (12), the stability of the closed-loop system obtained by the dashed lines in Fig. 1(b) can be analyzed by applying the GNC. The eigenvalue GNC variant [10] is closest in spirit to the $\mathrm{NC}$ and involves evaluating the eigenvalues $\lambda(s)$ of the matrix return ratio $\boldsymbol{G}(s)$. Plotting them for $s=j \omega$ gives the so-called characteristic loci, which, taken together, form the Nyquist curve. Solving 
for $\lambda(s)$ in the characteristic equation $|\lambda(s) I-\boldsymbol{G}(s)|=0$, where $I$ is the identity matrix, yields

$$
\begin{aligned}
\lambda(s) & =G_{d}(s) \pm \sqrt{\tilde{\mathbf{G}}(s) \tilde{\mathbf{G}}^{*}(s)-G_{q}^{2}(s)} \\
& =G_{d}(s) \pm j \underbrace{j \underbrace{G_{q}(s)}_{q(s) \sqrt{1-\frac{\tilde{\mathbf{G}}(s) \tilde{\mathbf{G}}^{*}(s)}{G_{q}^{2}(s)}}}}_{G_{q}^{\prime}(s)} .
\end{aligned}
$$

Since $\tilde{\mathbf{G}}(s) \tilde{\mathbf{G}}^{*}(s)$ has real coefficients, so has $G_{q}^{\prime}(s)$. Let $\lambda_{1,2}(s)$, respectively, correspond to the plus and minus signs in (34). We have $\lambda_{1}(-j \omega)=G_{d}(-j \omega)+j G_{q}^{\prime}(-j \omega)=$ $G_{d}^{*}(j \omega)+j G_{q}^{\prime *}(j \omega)=\left[G_{d}(j \omega)-j G_{q}^{\prime}(j \omega)\right]^{*}$. This is the mirror image in the real axis of $\lambda_{2}(j \omega)=G_{d}(j \omega)-j G_{q}^{\prime}(j \omega)$. Consequently, instead of evaluating both characteristic loci for $\omega>0$, it is sufficient to evaluate $\lambda_{1}(j \omega)$, i.e., the plus sign in (34), for $-\infty<\omega<\infty$-the same crossings of the Nyquist curve with the real axis are obtained, but symmetry about the real axis is lost. For this sake, bold-letter notation is used in the following equivalent expression for $\lambda_{1}$ :

$$
\lambda(s)=\mathbf{G}(s)+j G_{q}(s)\left[\sqrt{1-\frac{\tilde{\mathbf{G}}(s) \tilde{\mathbf{G}}^{*}(s)}{G_{q}^{2}(s)}}-1\right] .
$$

This expression is instructive, as it involves adding a term to $\mathbf{G}(s)$, i.e., the return ratio of the inner loop in Method 1. The mentioned term vanishes for $\tilde{\mathbf{G}}(s)=0$. Unlike Method 1 , it is sufficient to plot one Nyquist curve, that for $\lambda(s)$.

\section{Method 3 (Determinant GNC Variant Applied to the MIMO Model)}

Here, stability is verified if the Nyquist curve for the determinant of the return difference $I+\boldsymbol{G}(s)$ does not encircle the origin [11]. This is equivalent to the avoidance of the encirclement of -1 by the Nyquist curve for

$$
\gamma(s)=|I+\boldsymbol{G}(s)|-1 .
$$

We immediately obtain

$$
\begin{aligned}
\gamma(s) & =[1+\mathbf{G}(s)]\left[1+\mathbf{G}^{*}(s)\right]-\tilde{\mathbf{G}}(s) \tilde{\mathbf{G}}^{*}(s)-1 \\
& =\mathbf{G}(s)+\mathbf{G}^{*}(s)+\mathbf{G}(s) \mathbf{G}^{*}(s)-\tilde{\mathbf{G}}(s) \tilde{\mathbf{G}}^{*}(s) .
\end{aligned}
$$

As in Method 2, an addition to $\mathbf{G}(s)$ is made, but it is such that $\gamma(s)$ has real coefficients; hence, its notation is in italics.

Remark 6: Interestingly, (37) can intuitively be derived from Fig. 4(b) as follows. The characteristic equation for the closedloop system is given by $1+G_{s}(s)=1-\mathbf{G}_{a}(s) \mathbf{G}_{a}^{*}(s)=0$, with $\mathbf{G}_{a}(s)=\tilde{\mathbf{G}}(s) /[1+\mathbf{G}(s)]$

$$
1-\frac{\tilde{\mathbf{G}}(s) \tilde{\mathbf{G}}^{*}(s)}{[1+\mathbf{G}(s)]\left[1+\mathbf{G}^{*}(s)\right]}=0 .
$$

Multiplying by $[1+\mathbf{G}(s)]\left[1+\mathbf{G}^{*}(s)\right]$ now yields $1+\gamma(s)=0$, with $\gamma(s)$ given by (37).

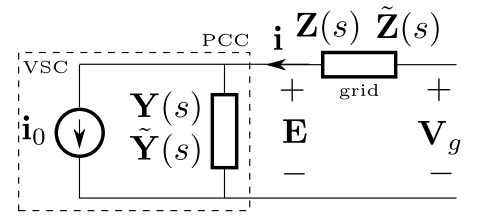

Fig. 5. VSC with asymmetric input admittance connected to a grid with asymmetric impedance.

\section{Application to Stability Analysis of Grid-Connected VSCs}

Connecting a VSC, with input-admittance parts given by (19) and (20), to an asymmetric grid, with impedance parts $\mathbf{Z}(s)$ and $\tilde{\mathbf{Z}}(s)$, gives the circuit model shown in Fig. 5. The grid voltage $\mathbf{V}_{g}$ is considered constant. Since the VSC input admittance is obtained by linearization and is operating-pointdependent, a constant current source $\mathbf{i}_{0}$ is added to $\mathbf{i}$. The circuit is represented by the complex-vector SISO model

$$
\begin{aligned}
\mathbf{i} & =\mathbf{Y}(s) \mathbf{E}+\tilde{\mathbf{Y}}(s) \mathbf{E}^{*}+\mathbf{i}_{0} \\
\mathbf{E} & =\mathbf{V}_{g}-\left[\mathbf{Z}(s) \mathbf{i}+\tilde{\mathbf{Z}}(s) \mathbf{i}^{*}\right] .
\end{aligned}
$$

This is an impedance-admittance cascade connection similar to (5) and (6), with the additions of $\mathbf{i}_{0}$ and $\mathbf{V}_{g}$. The transfer functions $\mathbf{G}(s)$ and $\tilde{\mathbf{G}}(s)$ of the cascade connection are obtained according to (8) and (9). Since $\mathbf{V}_{g}$ and $\mathbf{i}_{0}$ are constant, they do not impact the system stability. Considering $\mathbf{V}_{g}=\mathbf{i}_{0}=0$ reduces the closed-loop system to the SISO and MIMO models illustrated in Fig. 1 (dashed lines included).

1) Passive Grid Impedance: In many cases, the grid impedance is resistive-inductive-capacitive, implying that it is passive, i.e., it has a nonnegative passivity index for all frequencies. Then, as shown in [23], the Nyquist curve for the MIMO return ratio $\boldsymbol{G}(s)=\boldsymbol{Z}(s) \boldsymbol{Y}(s)$ cannot encircle -1 for $\omega$, where $p_{Y}^{\min }(\omega) \geq 0$. Consequently, in all three stability analysis methods under consideration, evaluation for $\omega$, where $p_{Y}^{\min }(\omega)<0$, is sufficient. In addition, for Method 1 , while a high sensitivity peak may lead to diminishing stability margins of the outer loop, it does not pose an instability risk if located, where $p_{Y}^{\min }(\omega) \geq 0$.

\section{NUMERICAL EXAMPLES}

To compare the three methods for the stability analysis of Section III, a VSC, as modeled in Section II-C, is considered. The grid impedance is considered as symmetric and, unless noted otherwise, purely inductive, $\mathbf{Z}(s)=\left(s+j \omega_{1}\right) L_{g}$, with $L_{g}=1$ per unit (p.u.), i.e., a short-circuit ratio of 1 . This represents a difficult operating condition for which instability phenomena easily occur.

For the converter filter, $L=0.1$ p.u., which is reasonable, at least for converters of higher power and voltage ratings. The CC, PLL, and DVC use pure P controllers, parameterized as

$$
\mathbf{F}_{c}(s)=\alpha_{c} L \quad F_{p}(s)=\frac{\alpha_{p}}{E_{0}} \quad F_{d}(s)=\frac{\alpha_{d}}{\kappa E_{0}}
$$

respectively, giving the closed-loop systems $\mathbf{G}_{c}(s)=\alpha_{c} /(s+$ $\left.\alpha_{c}\right), g_{p}(s)=\alpha_{p} /\left(s+\alpha_{p}\right)$, and $g_{d}(s)=\alpha_{d} /\left(s+\alpha_{d}\right)$. The PCC-voltage feedforward filter is selected as $\mathbf{H}(s)=\mathbf{G}_{c}(s)$. In the three examples that follow, $\alpha_{c}=5$ p.u., whereas $\alpha_{p}$, 

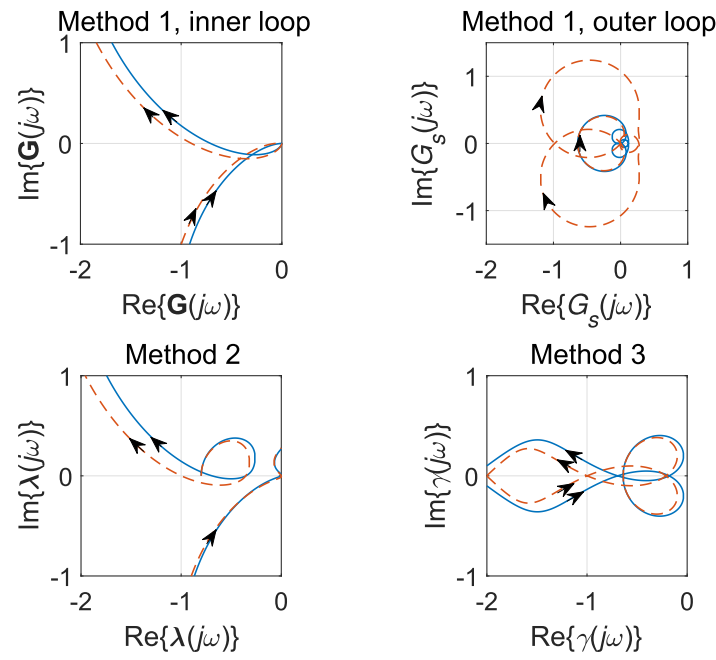

Fig. 6. Example 1: Nyquist curves for $\alpha_{d}=\alpha_{p}=0.4$ p.u. (solid) and $\alpha_{d}=\alpha_{p}=0.588$ p.u. (dashed).

$\alpha_{d}$, and the AVC are detailed later in the text. The operating points $E_{0}=1$ p.u. and $\mathbf{i}_{0}=i_{d 0}=0.8$ p.u. are considered. The latency is neglected, i.e., $\mathbf{G}_{l}(s)=1$, since the properties at higher frequencies are not studied.

\section{A. Example 1 (Identical PLL and DVC Dynamics, Without $A V C)$}

Here, $\mathbf{F}_{a}(s)=0$, while two different values of $\alpha_{d}=\alpha_{p}$ are considered: 0.4 and 0.588 p.u. The following observations can be made by inspecting Figs. 6 and 7 .

1) All three methods correctly show that $\alpha_{d}=\alpha_{p}=0.588$ p.u. gives the boundary to instability. For Method 1, the outer loop turns unstable.

2) Method 1 gives continuous Nyquist curves that are easy to interpret.

3) Method 2 results in a discontinuous Nyquist curve. A comparison indicates that this curve incorporates information from both curves of Method 1 .

4) Method 3 gives a convoluted Nyquist curve with numerous crossings of the negative real axis, making interpretation more difficult.

5) The top plot of Fig. 7 indicates that the root cause for the destabilization of the outer loop in Method 1 is the significant increase in the sensitivity peak obtained when changing $\alpha_{d}=\alpha_{p}$ from 0.4 to 0.588 p.u., accounting for the expansion of the Nyquist curve for the outer loop.

6) The bottom plot of Fig. 7 shows that the negativepassivity-index region about $\omega=0$ widens when $\alpha_{d}=$ $\alpha_{p}$ is increased from 0.4 to $0.588 \mathrm{p}$.u. This has the effect that the sensitivity peak falls into the negative-passivityindex region - an indication of stability problems [2].

To compare $\lambda(j \omega)$ as given by (35) with the characteristic loci $\lambda_{1,2}\{\boldsymbol{G}(j \omega)\}$ (all evaluated for $-\infty<\omega<\infty$ ), the latter are overlayed the former in Fig. 8(a), and the displayed area is enlarged relative to Fig. 6. As can be observed, both curves convey the same information, the latter with symmetry about the real axis.
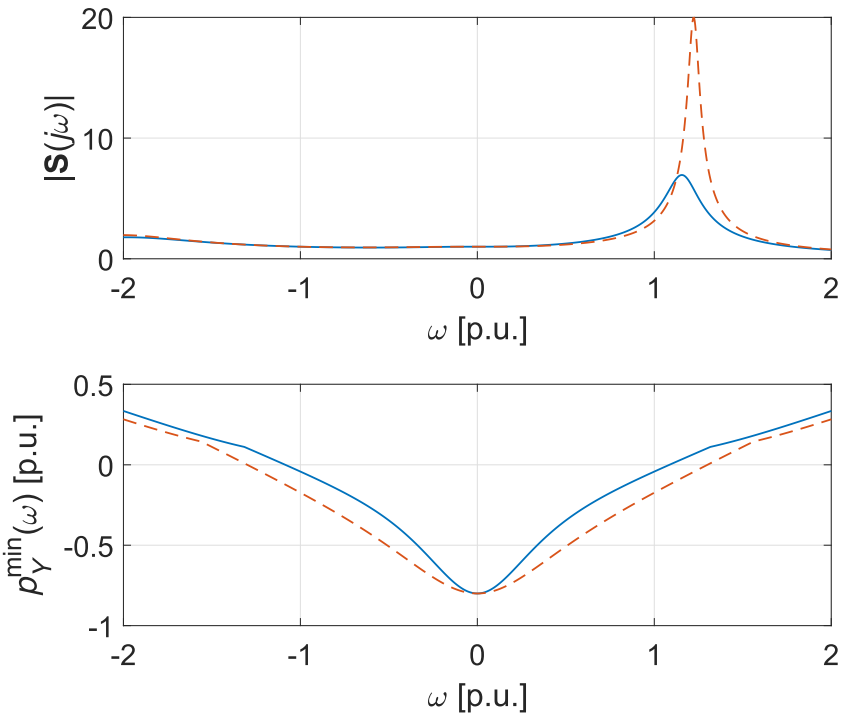

Fig. 7. Example 1: sensitivity function and input-admittance passivity index for $\alpha_{d}=\alpha_{p}=0.4$ p.u. (solid) and $\alpha_{d}=\alpha_{p}=0.588$ p.u. (dashed).
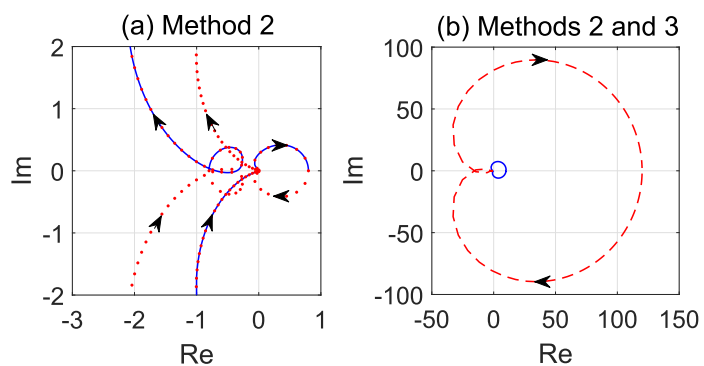

Fig. 8. Example 1: Nyquist curves for $\alpha_{d}=\alpha_{p}=0.4$ p.u. (a) $\lambda(j \omega)$ (solid) and characteristic loci of $\boldsymbol{G}(j \omega)$ (dotted). (b) $\lambda(j \omega)$ (solid) and $\gamma(j \omega)$ (dashed).

Fig. 8(b) depicts the full plots of $\lambda(j \omega)$ and $\gamma(j \omega)$, showing that the latter is much wider than the former; roughly $|\gamma(j \omega)| \sim|\lambda(j \omega)|^{2}$ for large $\omega$. This is the result of term $\mathbf{G}(s) \mathbf{G}^{*}(s)$ in (37), which causes the multiple crossings of the negative real axis as well.

\section{B. Example 2 (Different PLL and DVC Dynamics, With AVC)}

In this example, the claim of Remark 4 that the PLL can give a positive contribution to the passivity index for $i_{d 0}>0$ is put to test by letting $\alpha_{p}=1$ p.u. and $\alpha_{d}=0.1$ p.u. That is, the PLL is now ten times faster than the DVC. In addition, AVC with $F_{a}(s)=K_{a} \alpha_{a} /\left(s+\alpha_{a}\right), K_{a}=2$ p.u., (i.e., $\mathrm{P}$ with a low-pass filter) is included. Two different values of bandwidth $\alpha_{a}$ are used: 0.1 and 0.487 p.u., the latter which gives the boundary to instability. Comparing the solid curves in Fig. 9 with those in Fig. 6, increased stability margins can be observed. These can be correlated with the reduced width of the negative-passivity-index region in Fig. 10 relative to Fig. 7.

Increasing the bandwidth of the AVC filter gives instability (dashed curves). For Method 1, now the inner loop turns unstable (although the outer loop is close to being 

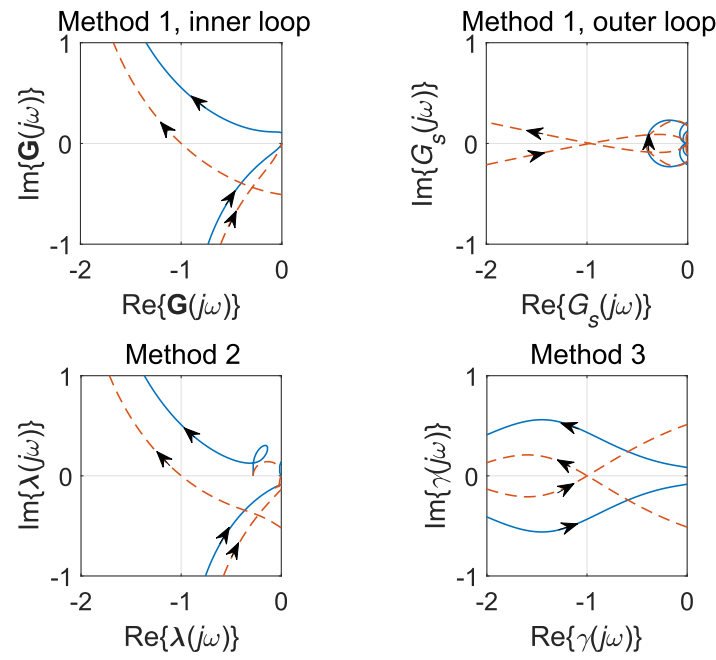

Fig. 9. Example 2: Nyquist curves for $\alpha_{a}=0.1$ p.u. (solid) and $\alpha_{a}=0.487$ p.u. (dashed).
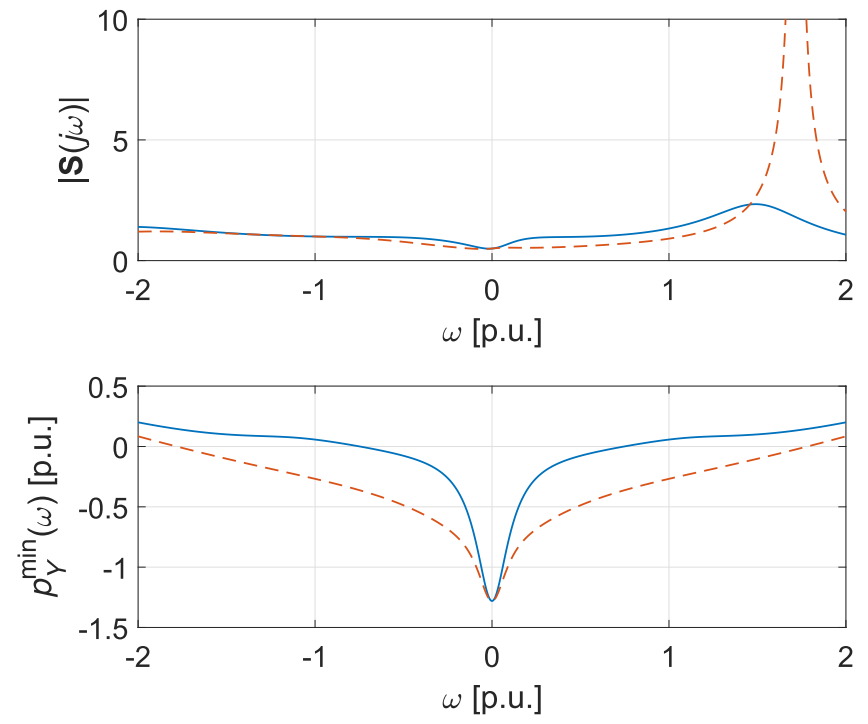

Fig. 10. Example 2: sensitivity function and input-admittance passivity index for $\alpha_{a}=0.1$ p.u. (solid) and $\alpha_{a}=0.487$ p.u. (dashed).

unstable, too). This results in an infinite sensitivity peak (see the top plot of Fig. 10).

\section{Example 3 (Identical PLL and DVC Dynamics, Without $A V C$ and With Grid Resonance)}

The inductive grid impedance is modified to an inductivecapacitive parallel impedance with the $\alpha \beta$-frame angular resonant frequency $\omega_{\text {res }}=1 /\left(L_{g} C_{g}{ }^{2}\right)$. For the control loops, $\alpha_{d}=\alpha_{p}=0.4$ p.u. and $F_{a}(s)=0$. Two different resonant frequencies are considered: 5.0 and 2.36 p.u.

In Fig. 11, all three methods immediately show stability for $\omega_{\text {res }}=5.0$ p.u. Methods 2 and 3 do so for $\omega_{\text {res }}=$ 2.36 p.u. as well. However, Method 1 too shows stability, since the dashed Nyquist curve for the inner loop encircles -1 clockwise, whereas that for the outer loop makes the encirclement counterclockwise. The outer loop stabilizes the unstable inner loop. This finding is of importance. Since
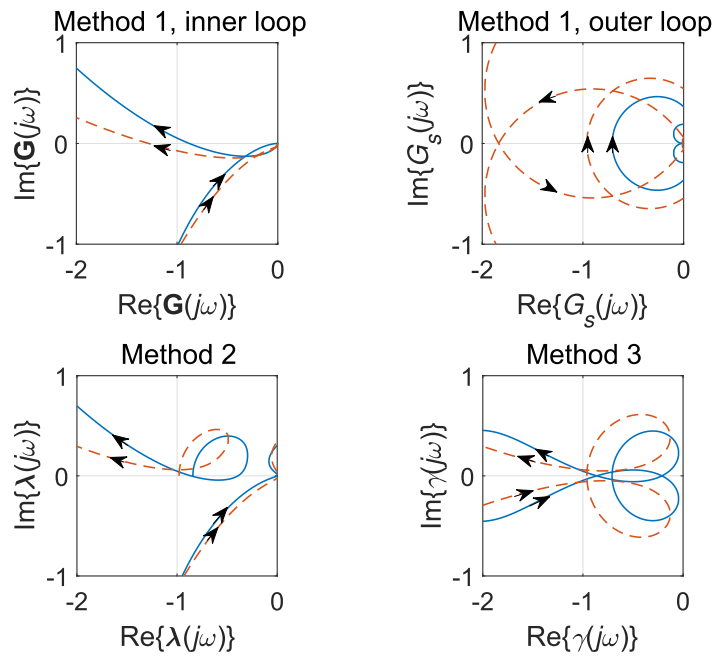

Fig. 11. Example 3: Nyquist curves for $\omega_{\text {res }}=5.0$ p.u. (solid) and $\omega_{\text {res }}=$ 2.36 p.u. (dashed).
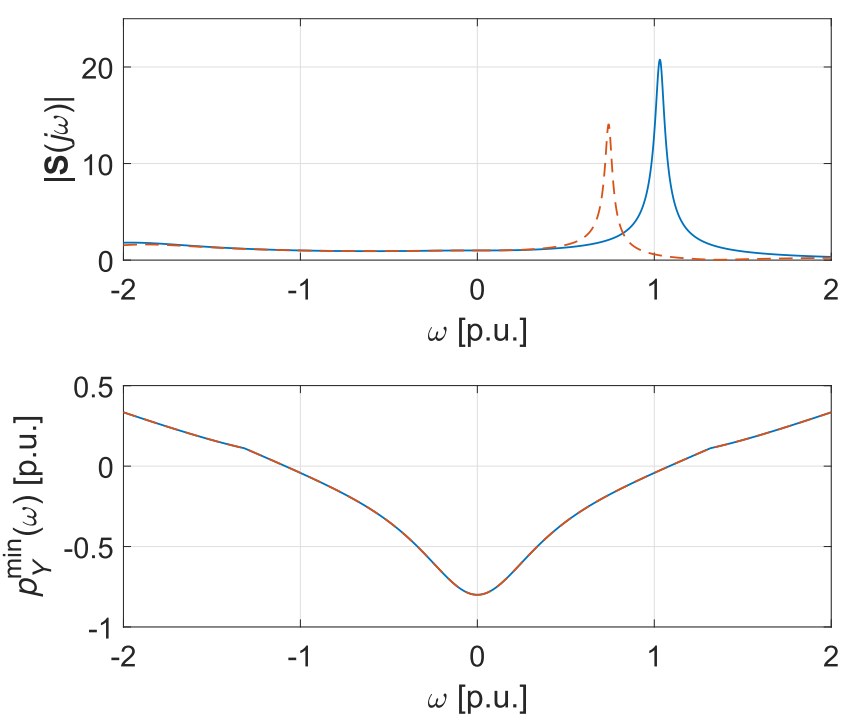

Fig. 12. Example 3: sensitivity function and input-admittance passivity index for $\omega_{\text {res }}=5.0$ p.u. (solid) and $\omega_{\text {res }}=2.36$ p.u. (dashed).

the PLL and DVC dynamics are identical, $\mathbf{Y}(s)$ is operatingpoint-invariant, whereas $\tilde{\mathbf{Y}}(s)$ is proportional to $-i_{d 0}$ [see (27) and (28)]. Consequently, the stability is conditional of the operating point $i_{d 0}=0.8$ p.u. For example, if instead $i_{d 0}=0$, then $\tilde{\mathbf{Y}}(s)=0 \Rightarrow \mathbf{G}_{a}(s)=0 \Rightarrow G_{s}(s)=0$, and the outer loop no longer stabilizes the inner loop.

The aggravated situation for the case $\omega_{\text {res }}=2.36$ p.u. can be correlated with the sensitivity peak coinciding with $p_{Y}^{\min }(\omega)<$ 0 [see Fig. 12]. Even though the sensitivity peak for $\omega_{\text {res }}=5.0$ p.u. is higher, it is merely on the boundary of the negativepassivity-index region.

1) Experimental Results: The case $\omega_{\text {res }}=2.36$ p.u. is verified experimentally using a VSC with ratings $7 \mathrm{kVA}, 200 \mathrm{~V}$ $(\mathrm{rms})$, and $50 \mathrm{~Hz}$. The sampling and switching frequencies are both $10 \mathrm{kHz}$, using a dSPACE DS1007 system where voltage and current measurement is made by a DS2004 high-speed analog-to-digital board. Gate signals are generated using a 


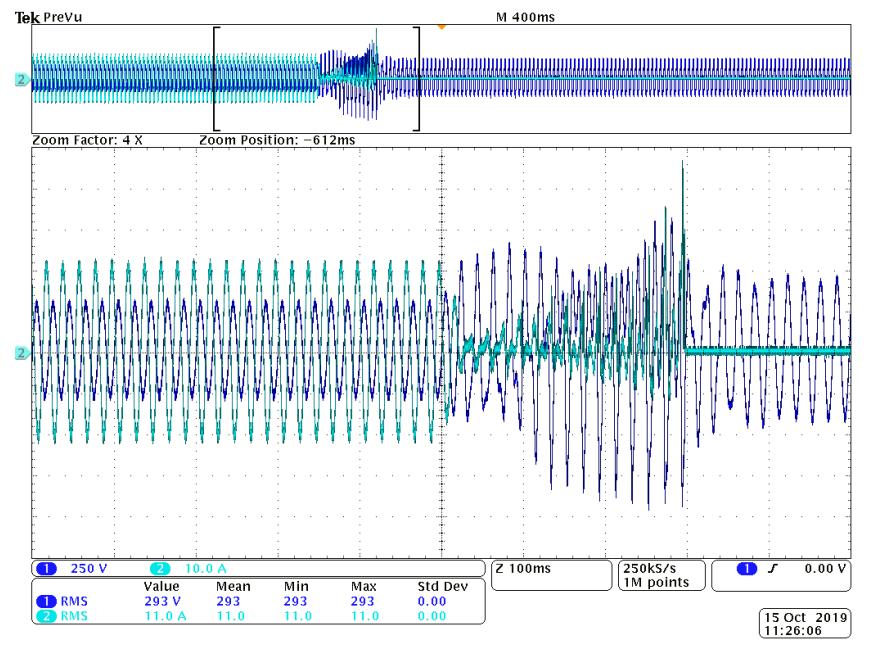

Fig. 13. Example 3: experimental verification for $\omega_{\text {res }}=2.36$ p.u. In the center of the displayed time interval, $P_{\mathrm{dc}}$ is zeroed, resulting in $i_{d 0}=0$ and instability.

DS5101 digital waveform output board. The parameters are identical to those of the theory; in addition, there are obviously losses in the circuit that are not accounted for in the model. Fig. 13 shows the PCC phase voltage and the converter phase current, for the same phase. (The curve of the latter, which initially has the larger amplitude, is shown with a sign change.) As can be observed, initially, where the dc-link load power $P_{\mathrm{dc}}$ is set so as to give $\mathbf{i}=0.8$ p.u., the system is stable. In the center of the displayed time interval, $P_{\mathrm{dc}}$ is zeroed, resulting in the current decaying to zero. In accordance with the finding of Method 1 that the stability is conditional of the operating point $i_{d 0}=0.8$ p.u., this results in instability and subsequent tripping of the converter.

Remark 7: In [12], it is argued that stability often can be (approximately) assessed by considering just the symmetric part of Fig. 1(a), i.e., the inner loop of Method 1. This is corroborated by the finding in Section III that the stability margins of the inner loop, via the sensitivity function $\mathbf{S}(s)$, affect the outer loop as well. In all three examples shown here, when at or close to instability (dashed curves), indeed the Nyquist curves for both loops show very poor stability margins. Examining the inner loop only may give results that are either slightly optimistic (as in Example 1), exact (as in Example 2), or slightly pessimistic (as in Example 3) concerning stability. Yet, it is advisable to examine both loops, as there may be cases where the results deviate from these findings.

\section{CONCLUSION}

Modeling and analysis of asymmetric systems using complex-vector SISO and MIMO models were considered. Both models carry the same information and are, thus, complimentary. The main usage for the MIMO model is for calculating the results that cannot be obtained using the SISO model.

One benefit of the approach is that direct complex-vector modeling of asymmetric systems is facilitated, where the real- and imaginary-part operators give contributions to both the symmetric and antisymmetric parts.

Another benefit is that complex transfer functions can be used for closed-loop stability analysis-particularly as applied to the grid-connected VSCs-avoiding matrix manipulations. In this respect, three candidate methods for stability analysis were presented and evaluated.

Method 1 (two-loop representation) shows that the stability margins of the inner loop affect also those of the outer loop by the sensitivity function. Two Nyquist curves need to be plotted, yet it is in general easy to assess the stability. Exceptions are certain close-to-instability cases, where the curves for the inner and outer loops may encircle -1 , respectively, clockwise and counterclockwise.

In Method 2 (eigenvalue GNC variant), one Nyquist curve suffices. Its interpretation, including realistic stability margins, is straightforward.

Method 3 (determinant GNC variant), on the other hand, is less useful, since the resulting Nyquist curve often is convoluted with multiple crossings of the negative real axis.

\section{APPENDIX}

The input-admittance contributions of the CC, PLL, DVC, and AVC are here derived.

The effect of the $\mathrm{CC}$ can be calculated by combining the relation $\left(s+j \omega_{1}\right) L \mathbf{i}=\mathbf{E}-\mathbf{v}$ obtained from Fig. 2 with (15) and the latency model $\mathbf{v}=\mathbf{G}_{l}(s) \mathbf{v}_{\text {ref }}$, giving

$$
\mathbf{i}=\mathbf{G}_{c}(s) \mathbf{i}_{\mathrm{ref}}+\mathbf{Y}_{c}(s) \mathbf{E}
$$

where the expressions for the transfer functions are given in (21) and (22).

The PLL gives a dynamic impact by the $d q$ transformations of $\mathbf{i}^{s}$ and $\mathbf{E}^{s}$ as well as by the $\alpha \beta$ transformation of $\mathbf{v}_{\text {ref }}$. This impact is nonlinear, and the technique for its linearization is well studied in the literature [12]. In brief, parameterization in the operating points and perturbation quantities is made as $\mathbf{i}^{s}=e^{j \omega_{1} t}\left(\mathbf{i}_{0}+\Delta \mathbf{i}\right), \mathbf{E}^{s}=e^{j \omega_{1} t}\left(E_{0}+\Delta \mathbf{E}\right)$, and $\mathbf{v}_{\mathrm{ref}}^{s}=$ $e^{j \omega_{1} t}\left(\mathbf{v}_{0}+\Delta \mathbf{v}_{\text {ref }}\right)$ [since the PLL aligns the $d q$ frame along $\mathbf{E}^{s}, E_{0}$ is real; evaluating (15) statically gives $\mathbf{v}_{0}=E_{0}-$ $j \omega_{1} L \mathbf{i}_{0}$ ]. Similarly, $\theta=\omega_{1} t+\Delta \theta$, allowing the $d q$ and $\alpha \beta$ transformations to be linearized according to the principle $\mathbf{i}=e^{-j \theta} \mathbf{i}^{s} \approx(1-j \Delta \theta)\left(\mathbf{i}_{0}+\Delta \mathbf{i}\right) \approx \mathbf{i}_{0}+\Delta \mathbf{i}-j \mathbf{i}_{0} \Delta \theta$. This results in the substitutions

$$
\mathbf{i} \rightarrow \mathbf{i}-j \mathbf{i}_{0} \Delta \theta \quad \mathbf{E} \rightarrow \mathbf{E}-j E_{0} \Delta \theta \quad \mathbf{v}_{\mathrm{ref}} \rightarrow \mathbf{v}_{\mathrm{ref}}+j \mathbf{v}_{0} \Delta \theta
$$

to be made in (15) to account for the PLL impact. Solving for $\mathbf{i}$ results in the addition of $j\left[\mathbf{G}_{c}(s) \mathbf{i}_{0}-E_{0} \mathbf{Y}_{c}(s)\right] \Delta \theta$ to (42). Eliminating $\Delta \theta$ by substituting (43) in (16) yields $\omega_{1} t+\Delta \theta=$ $\left[F_{p}(s) \operatorname{Im}\left\{E_{0}+\Delta \mathbf{E}-j E_{0} \Delta \theta\right\}+\omega_{1}\right] / s$, from which $\Delta \theta$ is solved as

$$
\Delta \theta=g_{p}(s) \operatorname{Im}\{\Delta \mathbf{E}\} / E_{0}
$$

where $g_{p}(s)$, given in (26), represents the closed-loop PLL dynamics. Since $\operatorname{Im}\{\Delta \mathbf{E}\}=\operatorname{Im}\{\mathbf{E}\}=\left(\mathbf{E}-\mathbf{E}^{*}\right) /(2 j)$, accounting for the PLL impact implies adding $\mathbf{Y}_{p}(s)\left(\mathbf{E}-\mathbf{E}^{*}\right)$ to (42), where $\mathbf{Y}_{p}(s)$ is given in (23). 
To calculate the DVC impact, the converter is approximated as lossless. Fig. 2 yields the power balance $W_{\mathrm{dc}}=\left(P-P_{\mathrm{dc}}\right) / s$. Substituting this relation in (17) and expressing the result in perturbation quantities (where $\Delta W_{\mathrm{dc}}^{\mathrm{ref}}=\Delta P_{\mathrm{dc}}=0$ ) give

$$
\Delta i_{d}^{\mathrm{ref}}=-\frac{F_{d}(s)}{s} \Delta P .
$$

Linearizing the relation $P=\kappa \operatorname{Re}\left\{\mathbf{E i}^{*}\right\}$ for the active input power yields $\Delta P=\kappa \operatorname{Re}\left\{E_{0} \Delta \mathbf{i}^{*}+\mathbf{i}_{0}^{*} \Delta \mathbf{E}\right\}=\kappa \operatorname{Re}\left\{E_{0} \Delta \mathbf{i}+\right.$ $\left.\mathbf{i}_{0}^{*} \Delta \mathbf{E}\right\}$, from which $\Delta \mathbf{i}$ is eliminated by substituting (42) (with perturbation quantities), giving

$$
\Delta P=\kappa \operatorname{Re}\left\{E_{0} \mathbf{G}_{c}(s) \Delta \mathbf{i}_{\text {ref }}+\left[E_{0} \mathbf{Y}_{c}(s)+\mathbf{i}_{0}^{*}\right] \Delta \mathbf{E}\right\} .
$$

In this process, the PLL impact on $\Delta P$ is neglected, which for most purposes represents a minor approximation. The $\mathrm{CC}$ and the DVC are normally tuned so that the closedloop dynamics of $\mathbf{i}$ are much faster than those of $W_{\mathrm{dc}}$. Yet, owing to the control law (17), $i_{d}^{\text {ref }}$ evolves on the time scale of $W_{\mathrm{dc}}$. This motivates considering $\mathbf{G}_{c}(s)=1$ in (46), which yields the simplification $\operatorname{Re}\left\{E_{0} \mathbf{G}_{c}(s) \Delta \mathbf{i}_{\text {ref }}\right\}=E_{0} \Delta i_{d}^{\text {ref }}$. Now, by substituting (46) in (45), the following simple solution of $\Delta i_{d}^{\text {ref }}$ is obtained:

$$
\Delta i_{d}^{\mathrm{ref}}=-g_{d}(s) \operatorname{Re}\left\{\left[\mathbf{Y}_{c}(s)+\mathbf{i}_{0}^{*} / E_{0}\right] \Delta \mathbf{E}\right\}
$$

where $g_{d}(s)$ is given in (26). An equivalent expression for (47) is $\Delta i_{d}^{\text {ref }}=\mathbf{Y}_{d}(s) \Delta \mathbf{E}+\mathbf{Y}_{d}^{*}(s) \Delta \mathbf{E}^{*}$, where $\mathbf{Y}_{d}(s)$ is given in (24). Substitution in (42), dropping the distinction of perturbation quantities, it is found that the DVC impact is accounted for by adding $\mathbf{G}_{c}(s)\left[\mathbf{Y}_{d}(s) \mathbf{E}+\mathbf{Y}_{d}^{*}(s) \mathbf{E}^{*}\right]$ to (42).

For the AVC, in (18), $|\mathbf{E}| \approx \operatorname{Re}\{\mathbf{E}\}$, since the $d q$ frame is aligned with $\mathbf{E}^{s}$. Thus, (18) in perturbation quantities becomes $\Delta i_{q}^{\text {ref }}=-F_{a}(s) \operatorname{Re}\{\Delta \mathbf{E}\}$, so the AVC impact is found simply by adding $\mathbf{Y}_{a}(s)\left(\mathbf{E}+\mathbf{E}^{*}\right)$ to (42), where $\mathbf{Y}_{a}(s)$ is given in (25).

\section{REFERENCES}

[1] L. Harnefors, "Modeling of three-phase dynamic systems using complex transfer functions and transfer matrices," IEEE Trans. Ind. Electron., vol. 54, no. 4, pp. 2239-2248, Aug. 2007.

[2] L. Harnefors, M. Bongiorno, and S. Lundberg, "Input-admittance calculation and shaping for controlled voltage-source converters," IEEE Trans. Ind. Electron., vol. 54, no. 6, pp. 3323-3334, Dec. 2007.

[3] K. D. T. Ngo, "Low frequency characterization of PWM converters," IEEE Trans. Power Electron., vol. PE-1, no. 4, pp. 223-230, Oct. 1986.

[4] M. Belkhayat, Stability Criteria for AC Power Systems With Regulated Loads. West Lafayette, IN, USA: Purdue Univ., 1997.

[5] S. Hiti, D. Boroyevich, and C. Cuadros, "Small-signal modeling and control of three-phase PWM converters," in Proc. IEEE Ind. Appl. Soc. Annu. Meeting, Dec. 2002, pp. 1143-1150.

[6] Z. Liu, J. Liu, W. Bao, and Y. Zhao, "Infinity-norm of impedance-based stability criterion for three-phase AC distributed power systems with constant power loads," IEEE Trans. Power Electron., vol. 30, no. 6, pp. 3030-3043, Jun. 2015.

[7] B. Wen, D. Boroyevich, R. Burgos, P. Mattavelli, and Z. Shen, "Smallsignal stability analysis of three-phase AC systems in the presence of constant power loads based on measured d-q frame impedances," IEEE Trans. Power Electron., vol. 30, no. 10, pp. 5952-5963, Oct. 2015.

[8] B. Wen, D. Boroyevich, R. Burgos, P. Mattavelli, and Z. Shen, "Analysis of D-Q small-signal impedance of grid-tied inverters," IEEE Trans. Power Electron., vol. 31, no. 1, pp. 675-687, Jan. 2016.

[9] T. Roinila, T. Messo, and E. Santi, "MIMO-identification techniques for rapid impedance-based stability assessment of three-phase systems in DQ domain," IEEE Trans. Power Electron., vol. 33, no. 5, pp. 4015-4022, May 2018.
[10] I. Postlethwaite and A. G. J. MacFarlane, A Complex Variable Approach to the Analysis of Linear Multivariable Feedback Systems. Berlin, Germany: Springer-Verlag, 1979.

[11] S. Skogestad and I. Postlethwaite, Multivariable Feedback Control: Analysis and Design Chichester, U.K.: Wiley, 1996.

[12] L. Harnefors, X. Wang, A. G. Yepes, and F. Blaabjerg, "Passivitybased stability assessment of grid-connected VSCs-An overview," IEEE J. Emerg. Sel. Topics Power Electron., vol. 4, no. 1, pp. 116-125, Mar. 2016.

[13] A. Rygg, M. Molinas, C. Zhang, and X. Cai, "A modified sequencedomain impedance definition and its equivalence to the dq-domain impedance definition for the stability analysis of AC power electronic systems," IEEE J. Emerg. Sel. Topics Power Electron., vol. 4, no. 4, pp. 1383-1396, Dec. 2016.

[14] A. Rygg, M. Molinas, C. Zhang, and X. Cai, "On the equivalence and impact on stability of impedance modeling of power electronic converters in different domains," IEEE J. Emerg. Sel. Topics Power Electron., vol. 5, no. 4, pp. 1444-1454, Dec. 2017.

[15] C. Zhang, X. Cai, A. Rygg, and M. Molinas, "Sequence domain SISO equivalent models of a grid-tied voltage source converter system for small-signal stability analysis," IEEE Trans. Energy Convers., vol. 33, no. 2, pp. 741-749, Jun. 2018.

[16] C. Zhang, M. Molinas, A. Rygg, and X. Cai, "Impedance-based analysis of interconnected power electronics systems: Impedance network modeling and comparative studies of stability criteria," IEEE J. Emerg. Sel. Topics Power Electron., early access, 2019, doi: 10.1109/JESTPE.2019.2914560.

[17] X. Wang, L. Harnefors, and F. Blaabjerg, "Unified impedance model of grid-connected voltage-source converters," IEEE Trans. Power Electron., vol. 33, no. 2, pp. 1775-1787, Feb. 2018.

[18] H. Zhang, X. Wang, L. Harnefors, H. Gong, J.-P. Hasler, and H.-P. Nee, "SISO transfer functions for stability analysis of gridconnected voltage-source converters," IEEE Trans. Ind. Appl., vol. 55, no. 3, pp. 2931-2941, May 2019.

[19] H. Zhang, L. Harnefors, X. Wang, H. Gong, and J.-P. Hasler, "Stability analysis of grid-connected voltage-source converters using SISO modeling," IEEE Trans. Power Electron., vol. 34, no. 8, pp. 8104-8117, Aug. 2019.

[20] J. Undrill and T. Kostyniak, "Subsynchronous oscillations part 1Comprehensive system stability analysis," IEEE Trans. Power App. Syst., vol. PAS-95, no. 4, pp. 1446-1455, Jul. 1976.

[21] M. W. Degner and C. M. Wolf, "Transient analysis of asymmetric AC systems using complex vectors," in Proc. IEEE Int. Electric Mach. Drives Conf. (IEMDC), Miami, FL, USA, May 2017, pp. 1-8.

[22] S. Shah and L. Parsa, "Impedance modeling of three-phase voltage source converters in DQ, sequence, and phasor domains," IEEE Trans. Energy Convers., vol. 32, no. 3, pp. 1139-1150, Sep. 2017.

[23] J. Bao, P. L. Lee, F. Wang, and W. Zhou, "New robust stability criterion and robust controller synthesis," Int. J. Robust Nonlinear Control, vol. 8, no. 1, pp. 49-59, Jan. 1998.

[24] L. Harnefors, R. Finger, X. Wang, H. Bai, and F. Blaabjerg, "VSC input-admittance modeling and analysis above the Nyquist frequency for passivity-based stability assessment," IEEE Trans. Ind. Electron., vol. 64, no. 8, pp. 6362-6370, Aug. 2017.

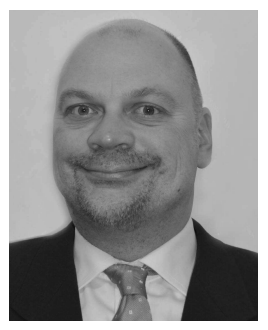

Lennart Harnefors (Fellow, IEEE) received the M.Sc., Licentiate, and Ph.D. degrees in electrical engineering from the Royal Institute of Technology (KTH), Stockholm, Sweden, in 1993, 1995, and 1997, respectively, and the Docent (D.Sc.) degree in industrial automation from Lund University, Lund, Sweden, in 2000

Between 1994 and 2005, he was with Mälardalen University, Västerås, Sweden, where he was a Professor of electrical engineering from 2001. Between 2001 and 2005, he was a part-time Visiting Professor of electrical drives with the Chalmers University of Technology, Göteborg, Sweden. In 2005, he joined the HVDC Product Group, ABB, Ludvika, Sweden, where he led the control development for cascaded-two-level-converter HVDC Light. In 2012, he also joined ABB, Corporate Research, Västerås, where he was appointed as a Senior Principal Scientist in 2013. In addition, he has been a part-time Adjunct Professor of power electronics with KTH since 2011. His research interests include control and dynamic analysis of power electronic systems, particularly grid-connected converters, and ac drives.

Dr. Harnefors was certified as a 2018 Outstanding Reviewer of the IEEE Transactions on Power Electronics. He is currently an Associate Editor of IET Electric Power Applications. 


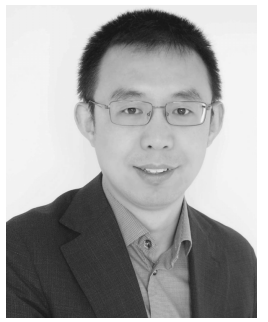

Xiongfei Wang (Senior Member, IEEE) received the B.S. degree in electrical engineering from Yanshan University, Qinhuangdao, China, in 2006, the M.S. degree in electrical engineering from the Harbin Institute of Technology, Harbin, China, in 2008, and the Ph.D. degree in energy technology from Aalborg University, Aalborg, Denmark, in 2013.

Since 2009, he has been with the Department of Energy Technology, Aalborg University, where he became an Assistant Professor in 2014, an Associate Professor in 2016, a Professor and the Research Program Leader of IEEE Electronic Power Grid (eGrid) in 2018, and the Director of the Huawei Energy Innovation Center in 2020. His current research interests include modeling and control of grid-interactive power converters, stability and power quality of converter-based power systems, and active and passive filters.

Dr. Wang serves as a Member-at-Large for the Administrative Committee of the IEEE Power Electronics Society (PELS) for the period of 20202022. He was selected into the Aalborg University Strategic Talent Management Program in 2016. He received the six IEEE Prize Paper Awards, the 2016 Outstanding Reviewer Award of the IEEE TransaCtIONS ON POWER ELECTRONICS, the 2018 IEEE PELS Richard M. Bass Outstanding Young Power Electronics Engineer Award, the 2019 IEEE PELS Sustainable Energy Systems Technical Achievement Award, and the 2019 Highly Cited Researcher by Clarivate Analytics (formerly Thomson Reuters). He serves as an Associate Editor for the IEEE TRANS ACTIONS ON POWER ELECTRONICS, the IEEE TRANSACTIONS ON INDUSTRY APPLICATIONS, and the IEEE JOURNAL OF EMERGING AND SELECTED TOPICS IN POWER ELECTRONICS.

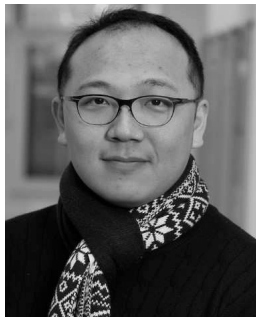

Shih-Feng Chou (Member, IEEE) received the B.S. and M.S. degrees in electrical engineering from National Tsing Hua University, Hsinchu, Taiwan, in 2009 and 2011, respectively. He is currently pursuing the Ph.D. degree with Aalborg University, Aalborg, Denmark.

He performed Research and Development in power electronics for renewable energy systems with Delta Electronics, Inc., Taoyuan, Taiwan, from 2012 to 2017. Since 2017, he has been a Research Assistant with the Department of energy technology, Aalborg University. His research interest focuses on modeling of large-scale power electronics-based power systems.

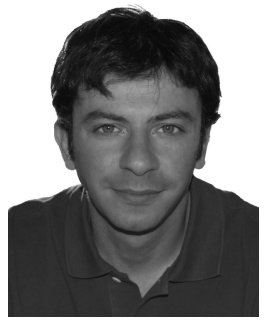

Massimo Bongiorno (Senior Member, IEEE) received the M.Sc. degree in electrical engineering from the University of Palermo, Palermo, Italy, in April 2002, and the Lic.Eng. and Ph.D. degrees from the Chalmers University of Technology, Gothenburg, Sweden, in December 2004 and September 2007, respectively.

From 2007 to 2010, he was an Assistant Professor with the Department of Electric Power Engineering, Chalmers University of Technology, where he became an Associate Professor in 2010. Since 2015, he has been holding the position of Professor in power electronic applications for power systems. His research interests include application of power electronics in power systems, power system dynamics, and power quality.

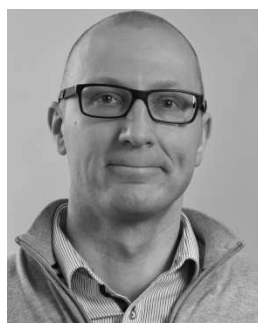

Marko Hinkkanen (Senior Member, IEEE) received the M.Sc.(Eng.) and D.Sc.(Tech.) degrees in electrical engineering from the Helsinki University of Technology, Espoo, Finland, in 2000 and 2004, respectively.

$\mathrm{He}$ is currently an Associate Professor with the School of Electrical Engineering, Aalto University, Espoo. His research interests include control systems, electric drives, and power converters.

Dr. Hinkkanen received the 2016 International Conference on Electrical Machines (ICEM) Brian J. Chalmers Best Paper Award and the 2016 and 2018 IEEE Industry Applications Society Industrial Drives Committee Best Paper Awards. He was the General Co-Chair of the 2018 IEEE 9th International Symposium on Sensorless Control for Electrical Drives (SLED). He is currently an Associate Editor of the IEEE TRANSACTIONS ON ENERGY CONVERSION and IET Electric Power Applications.

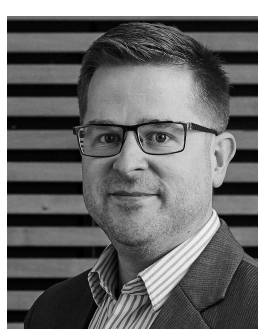

Mikko Routimo (Member, IEEE) received the M.Sc.(Eng.), Lic.Tech., and D.Sc.(Tech.) degrees in electrical engineering from the Tampere University of Technology, Tampere, Finland, in 2002, 2005, and 2009, respectively.

Since 2008, he has been with ABB Oy Drives, Helsinki, Finland, where he is currently a Principal Engineer. Since 2017, he has also been a Professor with the School of Electrical Engineering, Aalto University, Espoo, Finland. His research interests include power electronics in power systems, control of grid-connected converters, and electric drives. 